\title{
A Strain-Based Method to Estimate Longitudinal Force for Intelligent Tires by Using a Physics-Based Model
}

\author{
Haichao Zhou ${ }^{1}$-Huiyun Li1 ${ }^{1}$-Jian Yang $1,{ }^{*}$-Qingyun Chen ${ }^{1}$ - Guolin Wang ${ }^{1}$-Tong Han ${ }^{1}-$ Jieyu Ren ${ }^{2}-$ Te Ma $^{1}$ \\ 1 Jiangsu University, School of Automotive and Traffic Engineering, China \\ 2Zhejiang Wanxiang Marelli Shock Absorbers, China
}

Tires are essential components of vehicles and the only vehicle components in contact with the road. The tire longitudinal force originating from the contact between the tire and road can enhance traction and braking and contribute to the directional stability of vehicles. If the Iongitudinal tire force can be accurately estimated, vehicle safety can be improved. This study established a longitudinal tire physics-based model combining the brush model with the flexible ring model to develop a strain-based intelligent tire system for estimating the longitudinal tire force. The developed longitudinal dynamic model was used to study tire strain characteristics under pure longitudinal slip conditions. An algorithm was developed for estimating the longitudinal tire force through feature extraction and data fitting of the tire strain. A finiteelement tire model was established to simulate the longitudinal force. Comparing the simulated and estimated forces indicated that the proposed algorithm can accurately predict the longitudinal force of intelligent tires and thus provide useful information to vehicle stability control systems.

Keywords: intelligent tire, tire model, strain analysis, longitudinal force, force estimation

\section{Highlights}

- A longitudinal tire physics-based model combining the brush model with the flexible ring model is established and validated.

- Tire deformation characteristics under pure longitudinal slip conditions are analysed, and the effects of the longitudinal forces on radial deformation and circumferential strain are compared and analysed.

- The characteristic curve index of radial deformation and circumferential strain under different longitudinal forces are quantitatively analysed.

- A strain-based estimation algorithm for longitudinal tire force is proposed, and the simulation results verify the accuracy and credibility of the estimation algorithm.

\section{O INTRODUCTION}

Tires are the only parts of vehicles that come into contact with the road surface. The tire-road interaction directly affects tire performance and influences vehicle manoeuvrability [1]. The tireroad contact force can be determined by monitoring the interaction between tires and the road, and it can be used to improve the performance of stability controllers in intelligent vehicles [2]. This is why the automotive industry and scholars alike aim to estimate and control the forces accurately between tires and the road to enhance vehicle manoeuvrability and safety [3]. Intelligent tires can improve the safety of vehicles by providing information on tire force, tire load, road surface friction, and several other crucial contact characteristics [4].

Intelligent tires usually refer to tire systems equipped with sensors for monitoring tire dynamic parameters and road conditions to improve the dynamic control systems of vehicles. The sensor types used in such systems include accelerometers, piezoelectric, magnetic, and optical sensors; these sensors are selected for obtaining the surface acoustic waves, acceleration, deformation, and strain of tires [5]. Several intelligent tire systems have been proposed, and the results show that the intelligent tires provide a correlation between strain gauge measurements and tire operational conditions [6].

The most active and passive safety control systems are developed using a vehicle dynamic model, requiring the longitudinal tire forces to determine vehicle parameters, including longitudinal acceleration, braking distance, and slip rate [7]. Moreover, according to linear and nonlinear vehicle dynamic models, the longitudinal force acting on the tire is a major factor affecting the behaviour of a vehicle [8]. Thus, the estimation of the longitudinal force of intelligent tire has a great impact on vehicle handling and safety. By performing a polynomial fit, Yang et al. [9] estimated the longitudinal force using its relationship with the peak ratio between the tire's circumferential tensile strains and the front and rear contact areas. Matsuzaki and Todoroki [10] conducted data fitting for the functional relationship between the peak ratio of the tire circumferential compression strain in the contact patch and the braking torque of the front and rear wheels to monitor the braking behaviour of tires and estimate the longitudinal tire force. However, the function fitting process becomes 
complicated if several tire deformation features are extracted to assess the tire force. Consequently, the resultant fitting equation may have low accuracy.

Considering the complexity of tire structures and the coupling characteristics of tire forces during rolling conditions, machine-learning algorithms, such as neural network algorithms, are the most widely applied algorithms in tire force estimation. Zhao et al. [11] extracted acceleration signal features for different tire design schemes and estimated the vertical and longitudinal tire forces using a backpropagation neural network. Zhu and $\mathrm{Lu}$ [12] used a camera to track the displacement of tread and proposed a neural network algorithm to estimate tire force. However, when estimating tire forces by using machinelearning algorithms, the computing accuracy of the algorithms not only depends largely on the amount and scope of the training data but also requires the use of experimental tests and a simulation model to compute the variables. Developing a comprehensive intelligent-tire-based algorithm is a time-consuming and expensive process. Experimental data on velocity, tire pressure, various road conditions, and tire loading should be used to train the algorithm. The finite-element model (FEM) should be used to test tire component parameters properties, and it must be validated; therefore, FEM requires considerable computer resources.

Estimation algorithms based on tire physics-based models have received increasing attention due to high calculation efficiency and high estimation accuracy. The tire force is calculated according to a model of complex tire behaviors. Zhang and Yi [13] and Zhang et al. [14] developed a new type of pressure-sensitive conductive rubber sensor, and derived a formula for the tire-road friction force estimation based on the local friction force between the tire and road. Yi and Liang [15] implemented a polyvinylidene fluoride (PVDF)-based sensor to estimate tire deformation and motion, from a small vehicle model. They also presented an analytical sensing output model that can be used to capture the wheel/ground friction characteristics. Based on the test results of strain, Garcia-Pozuelo et al. [16] used a fuzzy logic algorithm to estimate tire slip angle and working conditions. Xiong and Tuononen [17] compared the carcass deformation of the flexible ring model with the measured results obtained using an optical laser sensor and proved that the tire flexible ring tire model can be used to predict tire deformation. Garcia-Pozuelo et al. [18] proposed a real-time physical model that reflects the longitudinal dynamics of intelligent tires and analysed the correlation between the measured tire strain and developed physical model to prove that the flexible ring tire model can be used to predict the strain on the inner liner. Thus, that model correlates strain information with dynamic tire parameters, predict changes in strain signals, and can be applied in the development of intelligent tire force estimation algorithms. APOLLO project claimed that strain sensors have merit over accelerometers for wheel force estimation. Also, the noise level of strain sensors is generally lower than the accelerometer noise and measured value is not affected by the tire rotational speed.

In this study, a strain-based tire longitudinal force estimation algorithm based on the physicsbased model was developed. First, a longitudinal dynamic tire model was established based on the flexible ring model and brush model; Second, the signal characteristics of the loaded tire were analysed, and the relationships between the strain information and longitudinal force were established according to the flexible ring model to estimate the longitudinal force; Finally, the strain information obtained from the finite-element model was substituted into the proposed algorithm to estimate the longitudinal force. The simulated values of the longitudinal force agreed favourably with the estimated values; thus, the practicability and accuracy of the developed algorithm were verified.

\section{ANALYTICAL MODEL OF TIRE LONGITUDINAL FORCE ESTIMATION}

\subsection{Flexible Ring Tire Model}

In this study, the relationship between the strain information of the inner liner and tire dynamic parameters was established according to the flexible ring tire model [19], and the details of the model symbols are provided in the appendices. The flexible ring model provides theoretical support for the development of intelligent tire algorithms. According to differences in stiffness, a tire is simplified into four parts, as shown in Fig. 1., the tread band, tread rubber, sidewall, and wheel. The belt is modelled as a thin ring and meets an assumption of inextensible beams. The sidewall is simplified to radial and tangential springs with radial and tangential damping, respectively, expressing the influence of tire sidewall forces on the belt. The rim is modelled as a rigid circle with mass and inertia. To determine the tire velocity, the ring is represented in terms of cylindrical coordinates. Two reference systems are used to express the dynamic behaviour of the ring, as depicted in Fig. 2. One 
system is a rotating reference system, which is a local coordinate system; the other system is a fixed reference system, which is a global coordinate system.

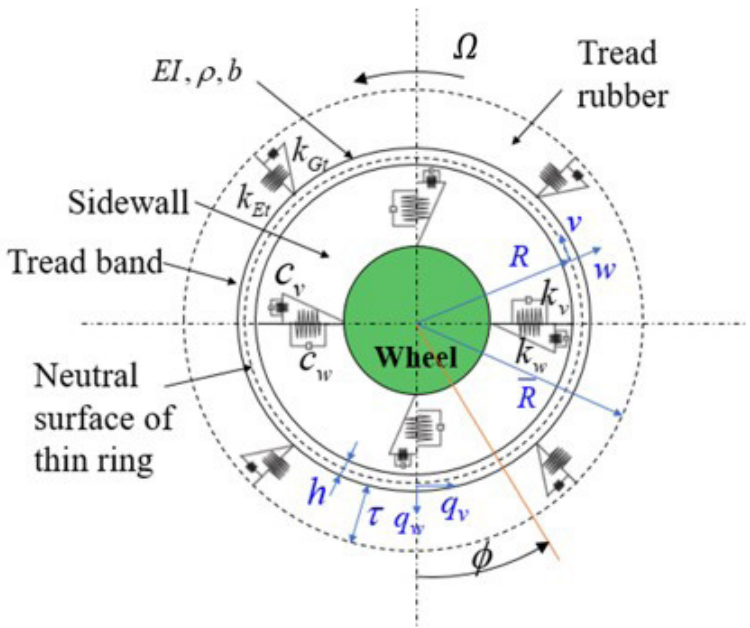

Fig. 1. Flexible ring tire model

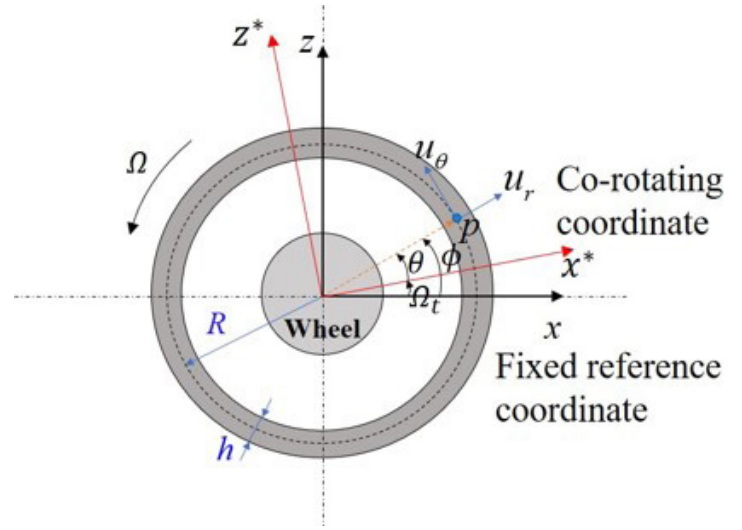

Fig. 2. Configurations of two tire coordinate systems

According to Hamilton's principle, the system reaches equilibrium when $\delta S=0$. The dynamic equation of the tire can be established as Eq. (1):

$$
\delta S=\int_{t_{1}}^{t_{2}}(\delta L+\delta W) d t,
$$

where $\delta W$ is the imaginary work of the nonconservative force, $L=T-V$ is the Lagrange function, $T$ is the kinetic energy of the system, and $V$ is the potential energy of the system.

According to Hamilton's principle, the following equation is obtained:

$$
\left\{\begin{array}{l}
L=T_{1}+T_{2}-S_{1}-S_{2} \\
\delta W=\delta E_{1}+\delta E_{2}
\end{array}\right.
$$

where $T_{1}$ is the kinetic energy of the ring, $T_{2}$ is the kinetic energy of the wheel, $S_{1}$ is the strain energy stored in the ring, $S_{2}$ is the elastic energy stored in the sidewall, and $\delta E_{1}$ and $\delta E_{2}$ represent the virtual work performed by extensional forces.

Because of the high extensional stiffness of the modern radial tire, the middle surface of the tire carcass is assumed to be inextensible, which means the circumferential length of the neutral surface of the ring is constant during the deformation. According to the Bernoulli-Euler assumption, the ring is an inextensible curved, bending beam [18]. Hence, the radial deformation and tangential deformation at any point on the middle surface of the elastic ring in a circumferential position satisfies $w=-\partial v / \partial \theta$. Under the condition that tire rolling is steady with no external moment acting on ring [20], the final equations of motion of the ring expressed in terms of the rotating coordinate system are derived as follows Eq. (3):

$-\frac{E I}{R^{4}}\left(\frac{\partial^{2} v}{\partial \theta^{2}}+2 \frac{\partial^{4} v}{\partial \theta^{4}}+\frac{\partial^{6} v}{\partial \theta^{6}}\right)+\frac{\sigma_{\theta}^{0} A}{R^{2}}\left(v+2 \frac{\partial^{2} v}{\partial \theta^{2}}+\frac{\partial^{4} v}{\partial \theta^{4}}\right)$

$+\rho A\left(\ddot{v}-\frac{\partial^{2} \ddot{v}}{\partial \theta^{2}}-4 \Omega \frac{\partial \dot{v}}{\partial \theta}+\Omega^{2}\left(\frac{\partial^{2} v}{\partial \theta^{2}}-v\right)\right)-k_{w} \frac{\partial^{2} v}{\partial \theta^{2}}$

$+k_{v} v-c_{w} \frac{\partial^{2} \dot{v}}{\partial \theta^{2}}+c_{v} \dot{v}-\frac{p_{0} b}{R}\left(v+\frac{\partial^{2} v}{\partial \theta^{2}}\right)=q_{v}+\frac{\partial q_{w}}{\partial \theta}$,

where $\sigma_{\theta}^{0}$ is the initial stress in the tangential direction of the belt, caused by the inflation pressure; $A=b h$ is the cross-sectional area of the thin ring of the tire.

When using the modal expansion method to solve the tire dynamic equations [21], the equation of motion of the radial deformation $w$. At the angle $\phi$ of the neutral surface of the thin ring under the forces $Q_{w}$ and $Q_{v}$ can be obtained as Eq. (4), where $Q_{w}$ and $Q_{v}$ are the magnitudes of radial and tangential line forces acting at specified stationary angular coordinate $\phi_{0}$.

$w(\phi)=$

$\sum_{n=1}^{N} A_{n}\left[n Q_{w} \cos n\left(\phi_{0}-\phi+\gamma_{n}\right)-Q_{v} \sin n\left(\phi_{0}-\phi+\gamma_{n}\right)\right] .(4)$

\subsection{Tread Longitudinal Brush Model}

When a tire rolls on the road, the distribution of tire contact pressure in the contact patch is nonsymmetrical. The contact pressure is concentrated at a distance $\Delta$ in front of the contact centre, as displayed in Fig. 3. Compared with tire contact pressure experiment results, an arbitrary pressure distribution is used to describe the pressure distribution in the contact patch. To facilitate analysis of the ring model, the centre of the contact patch is defined as $0^{\circ}$. 


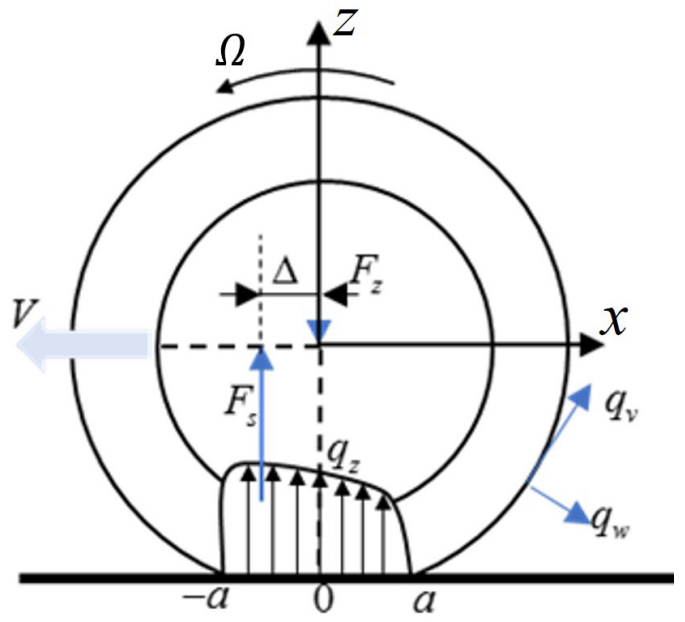

Fig. 3. Pressure distribution in the contact patch

According to the Unitire model [22], the normal pressure distribution $q_{z}(x)$ in the contact patch is expressed as follows Eq. (5):

$$
q_{z}(x)=\frac{F_{z}}{2 a} \cdot \eta\left(\frac{x}{a}\right)=\frac{F_{z}}{2 a} \cdot \eta(u),
$$

where $u=x / a$ is the relative coordinate in the contact patch, $2 a$ is the contact patch length, and $\eta(u)$ is the arbitrary pressure distribution function. If the vertical load offset is $\Delta, \quad \int_{-a}^{a} q_{z}(x) \cdot d x=F_{z} \quad$ and $\int_{-a}^{a} q_{z}(x) \cdot x \cdot d x=F_{z} \cdot \Delta$, then $\eta(u)$ should satisfy the following conditions:

$$
\left\{\begin{array}{l}
\eta(1)=\eta(-1)=0 \\
\eta(u) \geq 0, u \in[-1,1] \\
\eta(u)=0, u \notin[-1,1] \\
\int_{-1}^{1} \eta(u) d u=2 \\
\int_{-1}^{1} \eta(u) \cdot u \cdot d u=2 \cdot \frac{\Delta}{a}
\end{array} .\right.
$$

For the general tire loading conditions, $\eta(u)$ is expressed as Eq. (7):

$$
\eta(u)=A \cdot\left(1-u^{2 n}\right) \cdot(1-B u),
$$

According to the boundary conditions expressed in Eq. (6), $A$ and $B$ can be expressed as Eq. (8):

$$
\begin{gathered}
A=\frac{2 n+1}{2 n}, \\
B=-\frac{3 \cdot(2 n+3)}{2 n+1} \cdot \frac{\Delta}{a} .
\end{gathered}
$$

To determine the common contact pressure distribution of the radial tires, the following parameters are used: $n=2$ and $\Delta / a=0.04$. The contact pressure in contact patch is illustrated in Fig. 4.

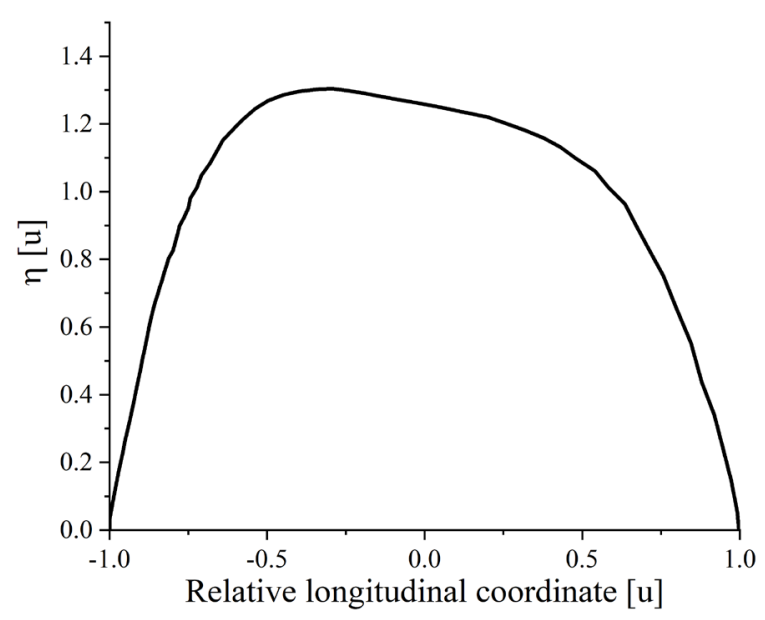

Fig. 4. Pressure distribution in contact patch

The rectangular coordinates of the pressure distribution function [Eq. (5)] are converted into angular coordinates [Eq. (10)] as follows:

$$
q_{z}(\phi)=\frac{F_{z}}{2 R\left|\phi_{f}\right|} \cdot \frac{5}{4}\left(1-u_{f}^{4}\right) \cdot\left(1+\frac{21}{125} \cdot u_{f}\right),
$$

where $\phi_{f}$ is the leading contact angle, $\phi$ is an arbitrary angle in the contact patch, and $u_{f}=\phi / \phi_{f}$.

For a loaded tire under longitudinal slip conditions, the lateral and camber characteristics are neglected, and the brush of the tread can only move in the longitudinal direction. The longitudinal slip ratio $S_{x}$ can be expressed as follows [23]:

$$
S_{x}=\frac{-V_{s x}}{\Omega R_{e}}=-\frac{V \cos \alpha-\Omega R_{e}}{\Omega R_{e}} \quad S_{x} \in(-\infty,+\infty),
$$

where $\Omega$ is the wheel angular speed; $R_{e}$ is the effective rolling radius, which is set as the mean radius of the ring $R ; V_{s x}$ is the longitudinal travel speed of the tire with respect to the road surface; $V$ is the wheel travelling speed; and $\alpha=0$ is the lateral angle.

The tire carcass is rigid, the tire elasticity is concentrated on the tread, the tread unit is replaced by a row of bristles, and the contact patch length of the bristles is $2 a$. With the development and advancement in the finite element method, the finite element analysis technology has been widely applied in the tire industry with high accuracy, and the contact patch length can be obtained using experimental test or finite element analysis. The contact patch length in this study was obtained using numerical simulation. To validate the tire FEM, the same tire, which is painted red, is tested on the self-developed tire test bench, as shown in Fig. 
5a. The vertical load is applied by moving the lead screw, and the sensor provides the vertical force in real time. The bottom part of the tire was painted with red ink and then pressed against a sheet of white paper, which was placed on the support plate. The grounding footprint from test and simulation are shown in Fig. $5 \mathrm{~b}$. The simulation results are in good agreement with the test results under the vertical force $4000 \mathrm{~N}$, and the maximum relative error of tire footprint was $1.74 \%$ in the contact patch width. This implied that tire grounding characteristics ensure that tire FEM model is accurate, and it can be used to verify the tire estimation algorithm of the longitudinal force in the subsequence analysis.

a)

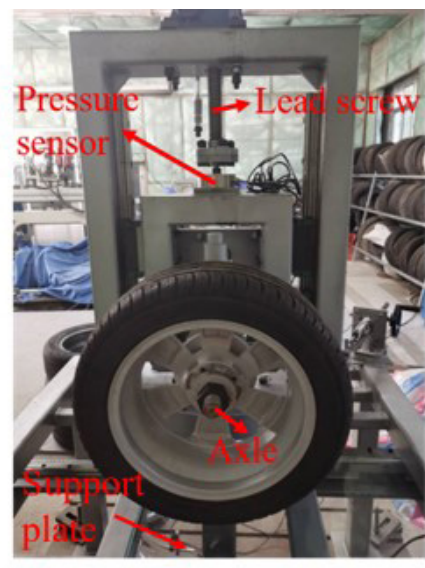

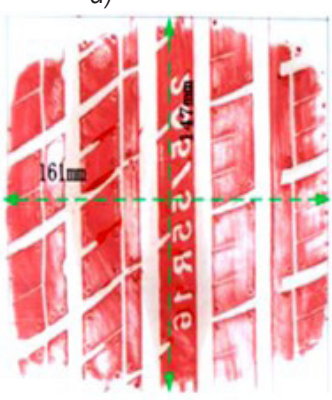

Test results

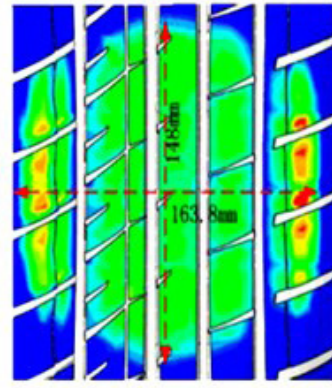

Simulation results b)

Fig. 5. Comparison of tire grounding footprint; a) tire stiffness test bench; and b) footprint parameters

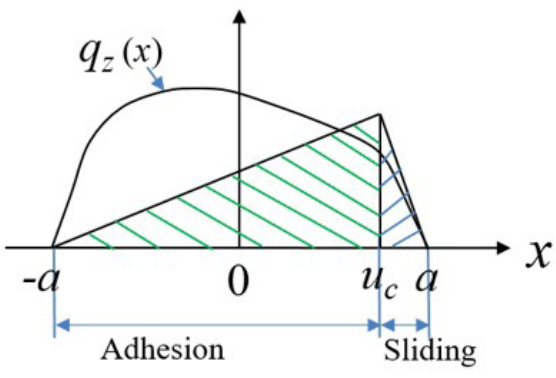

Fig. 6. Contact area of a tire brush model
Thus, the contact patch length of the bristles $2 a$ is $148 \mathrm{~mm}$. Due to the effect of the friction of the road surface, the grounding area of the brushes is divided into the adhesion region and slip region, as shown in Fig. 6.

Assuming that the longitudinal stress is caused by the deformation of the unit bristles in the contact patch, the longitudinal distribution force of the tread brush in the contact patch can be expressed as follows:

$$
q_{x}(x)=\left\{\begin{array}{c}
-(a-a u) k_{x} S_{x} \text { adhesion zone } \\
-\mu_{x} q_{z}(x) \operatorname{sgn}\left(S_{x}\right) \quad \text { slid zone }
\end{array},\right.
$$

where $k_{x}$ is the tangential stiffness of the bristle, which is set as the tread tangential stiffness $k_{G}$, and $\mu$ is the tire-road friction coefficient.

The split point $u_{c}$ divides the entire contact area into an adhesion zone and a sliding zone, and it is the sliding start point. When a sliding phenomenon occurs in the contact area, the split point $u_{c}$ can be derived from the Eq. (12):

$$
k_{x} \cdot(a-a u) S_{x}=\frac{\mu F_{z}}{2 a} \eta\left(u_{c}\right) .
$$

At this time, the total longitudinal force $F_{x}$ of tire in the contact patch is the sum of the longitudinal forces of two parts of the adhesion zone and slid zone as shown in Eq. (13).

$$
\begin{aligned}
F_{x} & =-\int_{-1}^{u_{c}} k_{x}(a-a u) \cdot S_{x} \cdot a d u \\
& -\left[\operatorname{sgn}\left(S_{x}\right)\right] \cdot \int_{u_{c}}^{1} q_{z}(u) \cdot \mu_{x} \cdot a d u .
\end{aligned}
$$

According to the coordinate transformation, the longitudinal force is expressed as a function of the angular coordinates as follows:

$$
q_{x}(\phi)=\left\{\begin{array}{ll}
-R\left(\left|\phi_{f}\right|-\phi\right) k_{x} S_{x}, & \text { Adhesion } \\
-\mu q_{z}(\phi) \operatorname{sgn}\left(S_{x}\right), & \text { Sliding }
\end{array} .\right.
$$

The force components of the neutral surface in the radial and tangential directions (Fig. 1) can be derived as follows:

$$
\left\{\begin{array}{l}
q_{w}(\phi)=-q_{z} \cos \phi+q_{x} \sin \phi \\
q_{v}(\phi)=q_{x} \cos \phi+q_{z} \sin \phi
\end{array} .\right.
$$

According to Eq. (14), the radial deformation and circumferential strain of the neutral surface are expressed as shown in Eqs. (15) and (16).

Where $N_{\phi}$ is the number of equally divided units of the tire tread in the contact patch, $\Delta \phi$ is the separation angle between every two adjacent tread units, and $q_{w}\left(\phi_{i}\right)$ and $q_{v}\left(\phi_{i}\right)$ are, respectively, the radial and tangential forces acting on the tire. 
The above work indicates that the strain and longitudinal force can be estimated by combining the flexible ring model with the brush model. Therefore, the estimation process of tire longitudinal force using the strain is illustrated in Fig. 7, and the application flow is subsequently described. Firstly, the tire contact angle of a loaded vertical force is obtained using the flexible ring model, and the vertical force distribution force is modelled with a parabolic function of the angular coordinate; Secondly, based on the brush tire model, the longitudinal force distribution is obtained with the slip ration and friction coefficient, and then, the longitudinal force distribution is divided into the radial and tangential directions; Lastly, the radial deformation and the circumferential strain of the ring are computed and obtained using Eqs. (15) and (16).

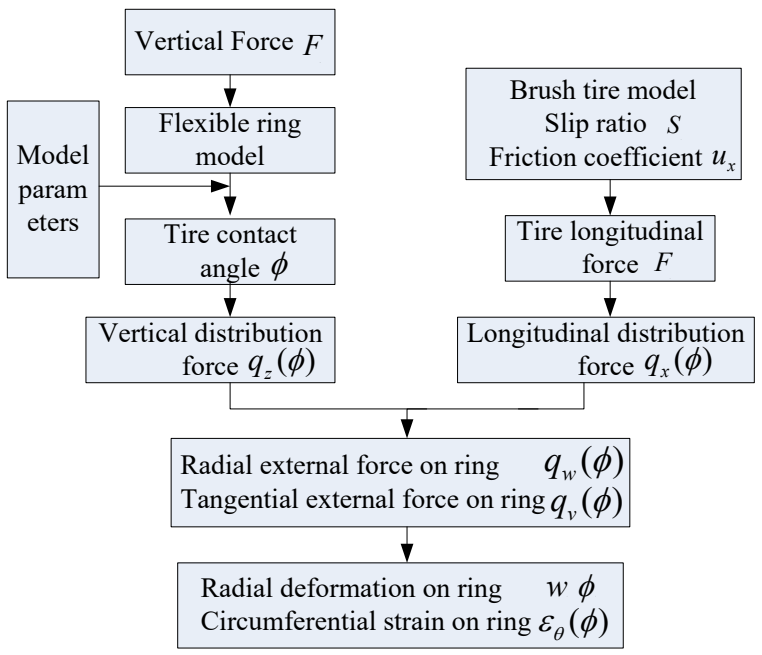

Fig. 7. Schematic of tire longitudinal force estimation

$$
\begin{gathered}
w(\phi)=\sum_{i=1}^{N_{\phi}} \sum_{i=1}^{\infty} n A_{n}\left[n q_{w}\left(\phi_{i}\right) \cos \left(n\left(\phi_{i}-\phi\right)\right)\right. \\
\left.-q_{v}\left(\phi_{i}\right) \sin \left(n\left(\phi_{i}-\phi\right)\right)\right] \cdot \Delta \phi, \\
\varepsilon_{\theta}(\phi)=-\frac{y}{R^{2}} \sum_{i=1}^{N_{\phi}} \sum_{n=1}^{\infty} n^{3} \cdot A_{n}\left[n q_{w}\left(\phi_{i}\right) \cos \left(n\left(\phi_{i}-\phi\right)\right)\right. \\
\left.-q_{v}\left(\phi_{i}\right) \sin \left(n\left(\phi_{i}-\phi\right)\right)\right] \cdot \Delta \phi .
\end{gathered}
$$

For a loaded tire, there is a contact length in the contact area, and the carcass and tread present deformation, as shown in the following Fig. 8, where $\phi_{f}$ and $\phi_{r}$ are the leading contact angle and the rear contact angle, respectively. The force distribution $q_{w}(\phi)$ caused by carcass deformation can be expressed as follows:

$$
q_{w}(\phi)=k_{E t} \cdot\left[-w(\phi)-d_{0}+\bar{R}(1-\cos \phi)\right] .
$$

Assuming just the vertical force is given, the force distribution $q_{w}(\phi)$ at both ends of the contact area is zero: $q_{w}\left(\phi_{f}\right)=q_{w}\left(\phi_{r}\right)=0$. Then the amount of sinking of the tire $d_{0}=R\left(1-\cos \phi_{f}\right)-w\left(\phi_{f}\right)$ is obtained. At the same time, within the tire contact area, the flexible model satisfies the following boundary conditions:

$$
\left\{\begin{array}{rl}
\chi_{1} & =\int_{\phi_{f}}^{\phi_{r}} q_{w}(\phi) d \phi-F_{z}=0 \\
\chi_{2} & =\left[w\left(\phi_{f}\right)-\bar{R}\left(1-\cos \phi_{f}\right)\right] . \\
& -\left[w\left(\phi_{r}\right)-\bar{R}\left(1-\cos \phi_{r}\right)\right]=0
\end{array} .\right.
$$

Based on the given model parameters of the flexible mode, the initial values of $\phi_{f}$ and $\phi_{r}$ are set, the radial deformation $w(\phi)$ and force distribution $q_{w}(\phi)$ can be obtained. The vertical force is estimated by integrating the force distribution within the contact angle. Taking the boundary conditions as criteria, if the error between the estimation of the vertical force and the given vertical force meets a demand, the contact angle $\phi$ is determined; otherwise, it needs to reset the values of $\phi_{f}$ and $\phi_{r}$, and recalculate $w(\phi)$ and $q_{w}(\phi)$. It will not stop until the error meets the demand that the contact angle is determined.

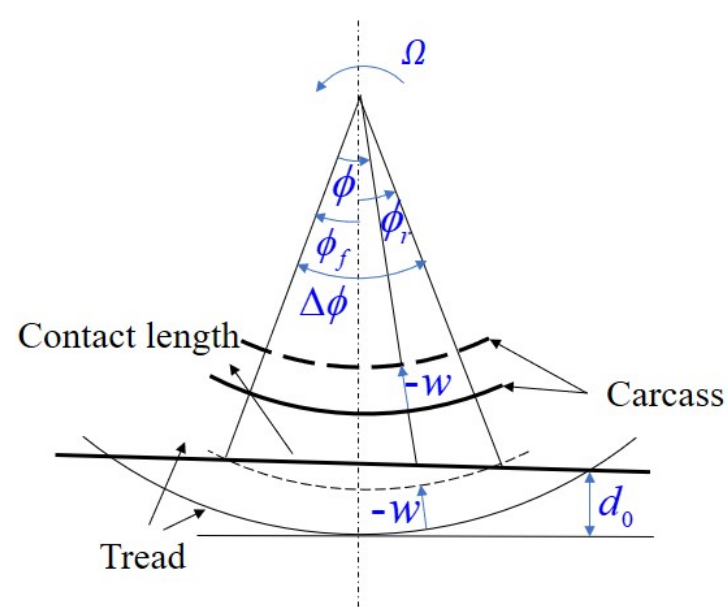

Fig. 8. Deformations of tread and carcass

\section{RESULTS AND DISCUSSION}

\subsection{Tire Model Analysis}

Through analysis of Eq. (4), the radial deformation of the neutral surface was discovered to be separable into the deformation caused by the tangential force and that caused by the radial force. When the tangential force 
is 0 , the radial deformation of the tire is approximately symmetrical; otherwise, the radial deformation of the tire is asymmetric.

Table 1. Flexible ring model parameters

\begin{tabular}{|c|c|c|c|c|c|}
\hline Parameter & Value & Unit & Parameter & Value & Unit \\
\hline$\rho$ & $1.8 \times 10^{3}$ & $\mathrm{~kg} / \mathrm{m}^{3}$ & $y$ & 0.0077 & $\mathrm{~m}$ \\
\hline$b$ & 0.185 & $\mathrm{~m}$ & $h$ & 0.00405 & $\mathrm{~m}$ \\
\hline$R$ & 0.306 & $\mathrm{~m}$ & $E I$ & 1.13 & $\mathrm{Nm}^{2}$ \\
\hline$k_{G t}$ & $5.4 \times 10^{6}$ & $\mathrm{~N} / \mathrm{m}^{2}$ & $k_{v}$ & $3.47 \times 10^{5}$ & $\mathrm{~N} / \mathrm{m}^{2}$ \\
\hline$k_{w}$ & $9.57 \times 10^{5}$ & $\mathrm{~N} / \mathrm{m}^{2}$ & $c_{w}=c_{v}$ & 0 & $\mathrm{Ns} / \mathrm{m}^{2}$ \\
\hline
\end{tabular}

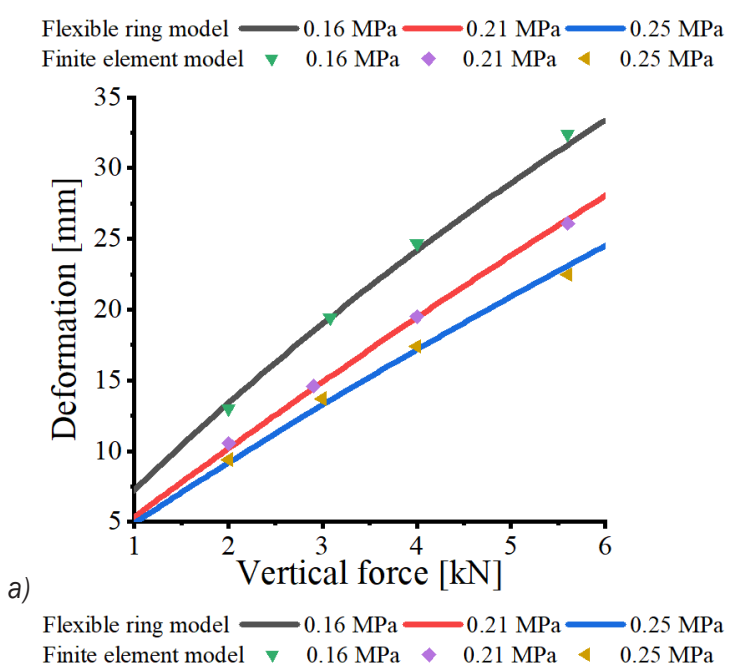

Finite element model $\nabla 0.16 \mathrm{MPa}-0.21 \mathrm{MPa} \& 0.25 \mathrm{MPa}$

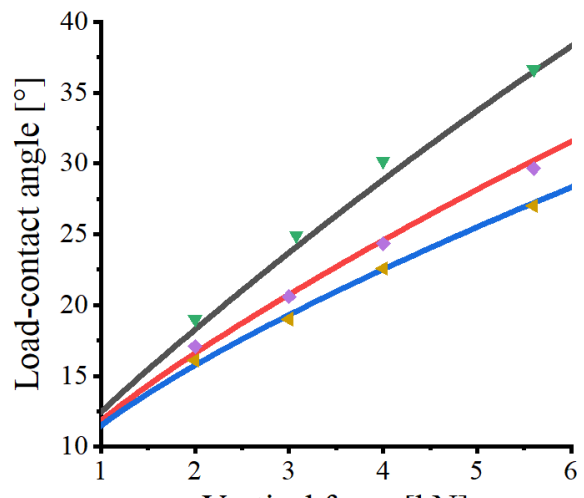

b)

Vertical force $[\mathrm{kN}]$

Fig. 9. Comparison of the results obtained using the finite-element method and flexible ring model; a) load-deformation; and b) load-contact angle

The detailed identification method for the flexible ring model is illustrated in [24]. Specifically, the geometric parameters of the flexible ring are obtained according to the cross-section of the pneumatic tire; the stiffness parameters are identified using modal test and load-sinkage test; the damping parameters are identified by varying the initial damping parameters and minimizing the error of contact angle between the simulated strains and the numerical ones using tire finite element analysis. The identified flexible ring model parameters are listed in Table 1. A comparison of the analytical results with the simulation results is shown in Fig. 9, which indicates the feasibility of the flexible ring model.

The model parameters presented in Table 1 indicate that the ring model can predict tire longitudinal forces when the vertical load is $4000 \mathrm{~N}$, the friction coefficient is 0.7 , the rolling velocity is $70 \mathrm{~km} / \mathrm{h}$, and the slip ratio is from $-20 \%$ to $20 \%$. Fig. 10 displays the longitudinal tire force calculated using Eq. (13) and the radial deformations of the neutral surface calculated using Eq. (15). It can be seen that when the longitudinal force is 0 , the deformations in the front and rear contact areas are approximately equal, and the radial deformation exhibits a symmetrical distribution. According to Fig. 3 , when the longitudinal force is positive, the radial deformation of the front contact area is smaller than that of the rear contact area. When the longitudinal force is negative, the deformation of the front contact area is larger than that of the rear contact area. Moreover, the larger the longitudinal force, the greater the asymmetry. It is noted that the longitudinal force has little effect on the radial deformation of the centre of the tire contact patch.

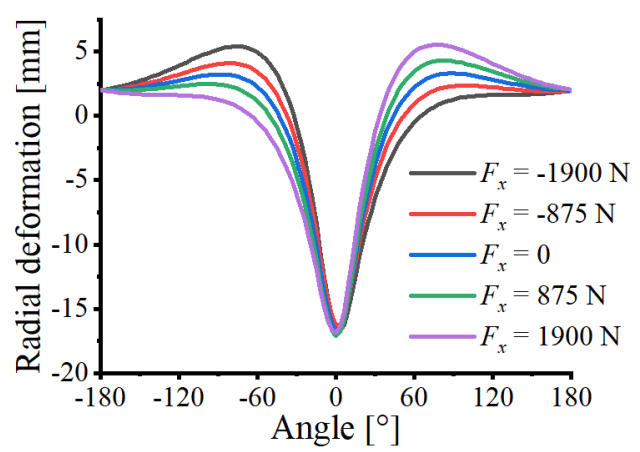

Fig. 10. Simulated deformation under different longitudinal forces with the vertical load $4000 \mathrm{~N}$

Xiong and Tuononen [17] utilized a laser-based tire sensor system for measuring tire deformation and compared the measured radial deformations under different longitudinal forces; the test results are shown in Fig. 11. As seen in Fig. 11, increasing tire longitudinal force will lead to decrease in the radial deformation of the front contact area and increase in the radial deformation of the rear contact area. Under the conditions that the longitudinal force $0 \mathrm{~N}, 875 \mathrm{~N}$ and $1900 \mathrm{~N}$, and comprehensive change trend analysis 
of the comparison between the simulation result in Fig. 10 and the measured result in Fig. 11, although vertical tire force is different, the radial deformation trends of simulation results were the same as the measured results, it can be seen that the flexible ring model established in the study is accurate and credible.

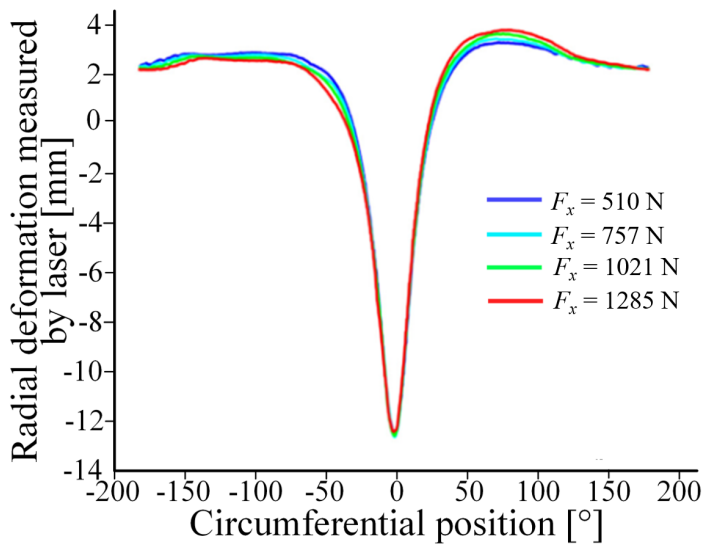

Fig. 11. Measured radial deformations under different longitudinal forces with the vertical load 3000 N [17]

The circumferential strain of the neutral surface, which is calculated using Eq. (16), is illustrated in Fig. 12. When the longitudinal force is 0 , the strain in the front and rear contact areas is approximately equal, and the strain curve displays symmetric distribution. When the longitudinal force is positive, the circumferential strain of the front contact area is less than that of the rear contact area. When the longitudinal force is negative, the circumferential strain of the front contact area is larger than that of the rear contact area. Thus, the longitudinal force causes asymmetric strain distribution in the front and rear areas of the tire. As the longitudinal force increases, the asymmetry of the strain curve also increases. However, the longitudinal force has almost no effect on the maximum tensile strain.

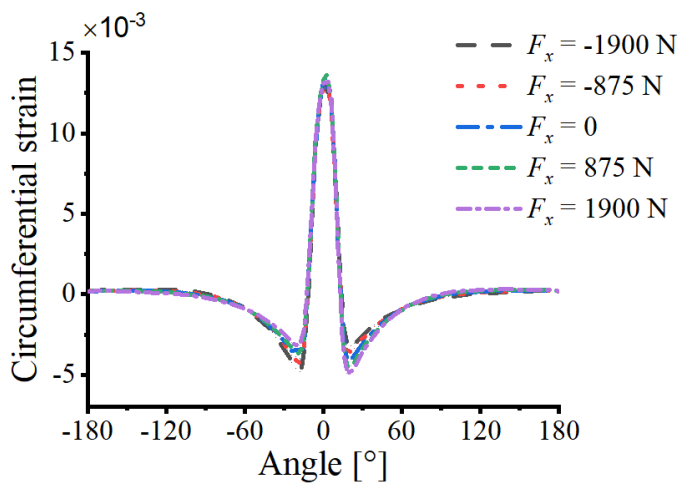

Fig. 12. Circumferential strain under different longitudinal forces
The variation in the circumferential strain distribution with the longitudinal force can be used to identify the driving conditions of the tire. The front and rear contacts of the tire are displayed in Fig. 13, in which the tire's rolling direction is considered the positive direction. The peak circumferential strain at the front contact is denoted by $C P_{f}$, and the peak circumferential strain at the rear contact is denoted by $C P_{r}$. The tire conditions can be defined using the different relationships between $C P_{f}$ and $C P_{r}$, as shown in the following travel state. Comprehensive analysis of the coordinate directions in Fig. 2 and the longitudinal forces in Fig. 12 indicates that the tire is in the driving condition when the longitudinal forces are negative and that the tire is in the braking condition when the longitudinal forces are positive.

$$
\left\{\begin{array}{lc}
C P_{f} \approx C P_{r} & \text { Free rolling } \\
C P_{f}<C P_{r} & \text { Braking } \\
C P_{f}>C P_{r} & \text { Driving }
\end{array} .\right.
$$

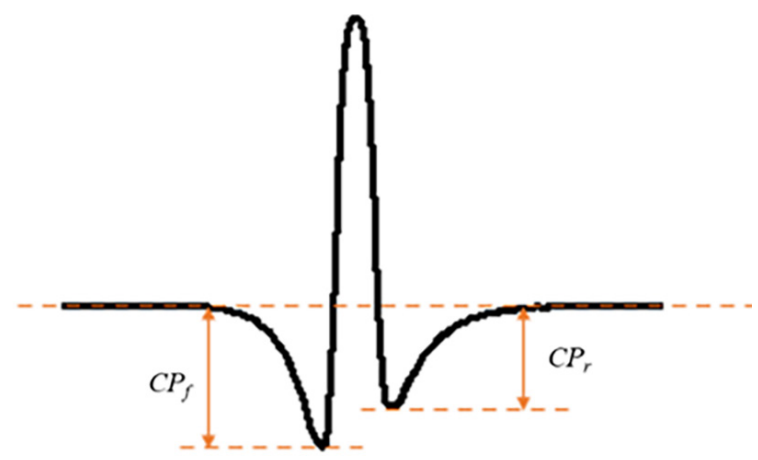

Fig. 13. Circumferential strain distribution of the neutral surface

\subsection{Characteristic Analysis of Radial Deformation}

To analyse the asymmetry in the neutral surface's radial deformation caused by the longitudinal force, the radial deformation is defined as follows. The $0^{\circ}$ coordinate position of the contact patch centre is taken as the midpoint along the rolling direction; thus, the front region comprises negative angles, and the rear region comprises positive angles (Fig. 14). The angle range in the front area is denoted $N_{\phi f}$, whereas that in the rear area is denoted $N_{\phi r}$. An angle $\phi_{j},(j=1, \ldots$, $\left.N_{\phi f}\right)$ in the front area corresponds to an angle $-\phi_{j}$, $\left(j=1, \ldots, N_{\phi r}\right)$ in the rear area. The radial deformation of the angle $\phi_{j}$ in the front area is denoted $w\left(\phi_{j}\right)$, and the radial deformation of the angle $-\phi_{j}$ in the rear area is denoted $w\left(-\phi_{j}\right)$. According to the radial deformation characteristics displayed in Fig. 4, if the longitudinal 
force is equal to $0, w\left(\phi_{j}\right)=w\left(-\phi_{j}\right)$. If the longitudinal force is not equal to $0, w\left(\phi_{j}\right) \neq w\left(-\phi_{j}\right)$. Therefore, the difference between $w\left(\phi_{j}\right)$ and $w\left(-\phi_{j}\right)$ (denoted as $\left.\Delta w\right)$ is used to characterize the degree of asymmetry of the radial deformation. According to the tire rolling direction, $\Delta w$ can be expressed as follows:

$$
\left\{\begin{array}{ll}
\Delta w\left(\phi_{j}\right)=w\left(\phi_{j}\right)-w\left(-\phi_{j}\right) & -\pi \leq \phi_{j} \leq 0 \\
\Delta w\left(-\phi_{j}\right)=w\left(-\phi_{j}\right)-w\left(\phi_{j}\right) & 0 \leq-\phi_{j} \leq \pi
\end{array} .\right.
$$

Eq. (19) indicates that the deformation differences in the front and rear areas have the same magnitude but in the opposite direction.

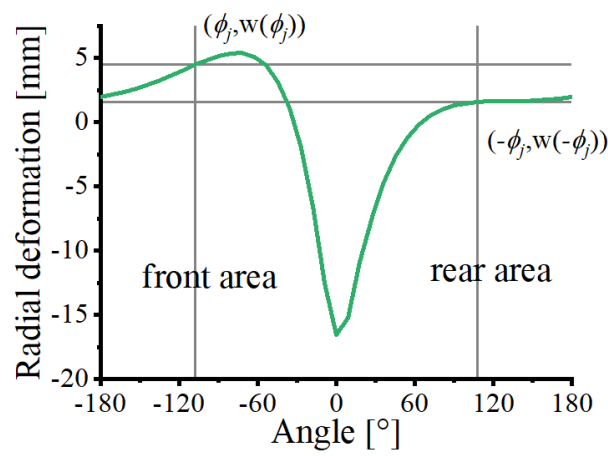

Fig. 14. Radial deformation of the ring neutral surface

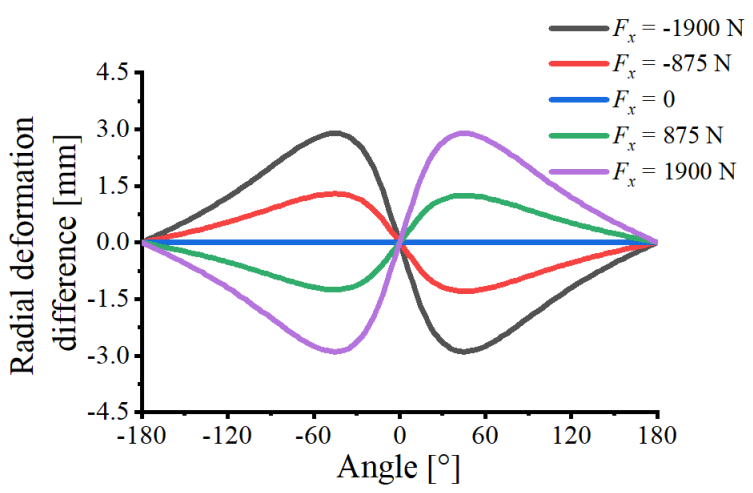

Fig. 15. Curves of $\Delta \omega$ under different longitudinal forces

To analyse the effect of the longitudinal force on the radial deformation difference $\Delta \omega$, the longitudinal dynamic model of the thin ring is analysed under a vertical load of $4000 \mathrm{~N}$, a friction coefficient of 0.7 , roll velocity of $70 \mathrm{~km} / \mathrm{h}$, and slip rate range of $-20 \%$ to $20 \%$. The analytical results are displayed in Fig. 15. When the longitudinal force is equal to 0 , the $\Delta \omega$ is also equal to 0 . When the longitudinal force is positive, the radial deformation difference in the front area is negative, and that in the rear area is positive. When the longitudinal force is negative, the radial deformation difference in the front area is positive, and that in the rear area is negative. As the longitudinal force increases, the change in the radial deformation difference curve increases.

\subsection{Analysis of the Circumferential Strain Characteristics}

To analyse the effect of the vertical force on the radial deformation difference under the same longitudinal force, the following boundary conditions were adopted: vertical load range of $2900 \mathrm{~N}$ to $5600 \mathrm{~N}$, friction coefficient range of 0 to 0.7 , and slip rate of $20 \%$. Because of the high slip rate, the tread brushes are in the slipping zone. The friction coefficient can be changed under different vertical forces to obtain the longitudinal force $(2000 \mathrm{~N})$. The effect of the vertical force on the radial deformation difference between the front and rear areas is displayed in Fig. 16. The results indicate that the vertical force has almost no influence on the radial deformation difference. Thus, the radial deformation difference between the front and rear areas is mainly affected by the longitudinal force.

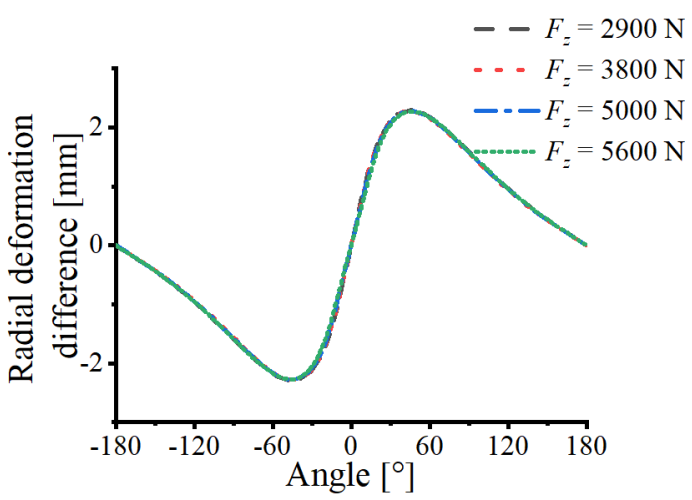

Fig. 16. Curves of $\Delta \omega$ under different vertical forces

The circumferential strain difference $\Delta \varepsilon_{\theta}$ between the front and rear regions can be defined by using the method for determining the radial deformation difference between these regions. The curves of circumferential strain difference under the same conditions as those used for analysing the radial deformation difference are displayed in Fig. 17. When the longitudinal force is equal to 0 , the difference in the circumferential strain of the front and rear areas is also 0 . When the longitudinal force is positive, the maximum value of the circumferential strain difference curve is in the front area and its minimum value is in the rear area. The variation of the circumferential strain difference increases with an increase in the longitudinal force.

The curves of circumferential strain difference for different vertical forces under the same longitudinal 
force are displayed in Fig. 18. The variation in the circumferential strain difference does not change significantly with an upsurge in the vertical force. Thus, the circumferential strain difference between the front and rear areas is mainly affected by the longitudinal force.

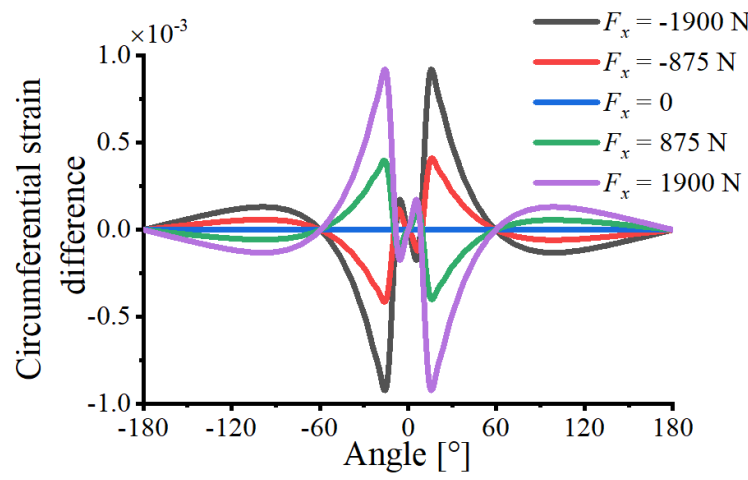

Fig. 17. Curves of $\Delta \varepsilon_{\theta}$ under different longitudinal forces

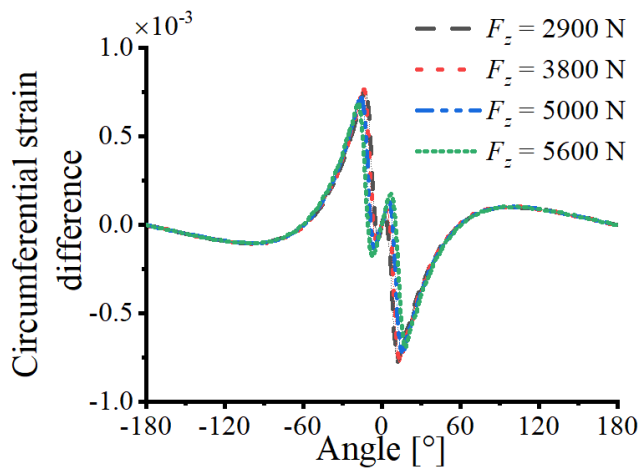

Fig. 18. Curves of $\Delta \varepsilon_{\theta}$ under different vertical forces

\subsection{Characteristic Curve Index}

The difference in the radial deformation and circumferential strain between the front and rear regions reflects the degree of asymmetry of the deformation and strain, respectively. To quantitatively analyse the relationship between the radial deformation difference or circumferential strain difference and the longitudinal force, this study employed the difference curve area, which was defined as the area under the curve obtained by setting the radial deformation difference or circumferential strain difference as the vertical coordinate and the tire angle as the horizontal coordinate. A tire is assumed to pass through point $P$ at equal intervals over one revolution; thus, the difference area of the tire revolution can be expressed as follows:

$$
A_{\omega}=\sum_{i=1}^{P} \Delta \omega\left(\phi_{i}\right) \Delta \phi, \quad A_{\varepsilon}=\sum_{i=1}^{P} \Delta \varepsilon_{\theta}\left(\phi_{i}\right) \Delta \phi,
$$

where $\Delta \phi$ is the interval angle between two discrete points, $A_{\omega}$ is the area of the difference curve of the radial deformation, and $A_{\varepsilon}$ is the area of the difference curve of the circumferential strain.

a)

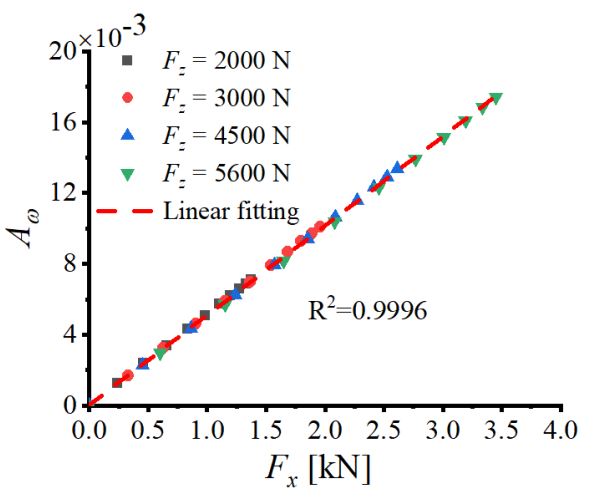

b)

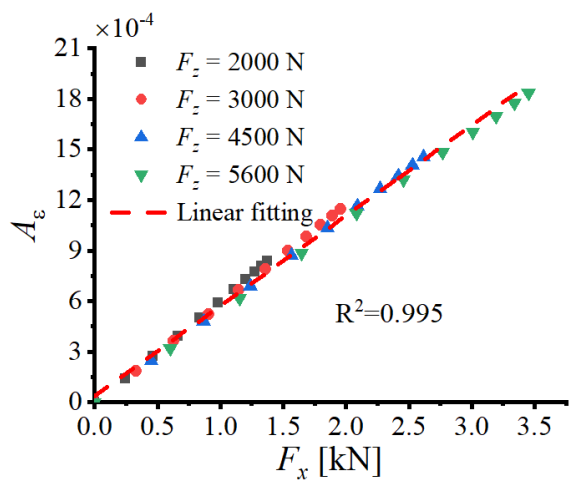

Fig. 19. Relationship between the difference curve area and longitudinal tire force; a) relationship between $A_{\omega}$ and $F_{x}$; and b) relationship between $A_{\varepsilon}$ and $F_{x}$

To quantitatively analyse the effect of the longitudinal force on the difference curve area, the difference curve area of the radial deformation difference and circumferential strain difference curves were calculated using Eq. (20) under the following conditions: friction coefficient of 0 to 0.7 , roll velocity of $70 \mathrm{~km} / \mathrm{h}$, vertical load range of 2000 $\mathrm{N}$ to $5600 \mathrm{~N}$, and slip rate range of $0 \%$ to $20 \%$. Fig. $19 \mathrm{a}$ indicates that $A_{\omega}$ has a linear relationship with the longitudinal force, and the correlation coefficient for this relationship is 0.9996 . Fig. $19 \mathrm{~b}$ indicates that $A_{\varepsilon}$ has a linear relationship with the longitudinal force, and the correlation coefficient for this relationship is 0.995. It can be inferred from Fig. 19 that the vertical force has little influence on $A_{\omega}$ and $A_{\varepsilon}$. Theoretically, the $A_{\omega}$ and $A_{\varepsilon}$ can be used as a tire signal to estimate tire longitudinal force. Nevertheless, previous research related to the intelligent tires indicates that the strain sensors meet the requirements to achieve and advance intelligent tire system by means of strain 
measurement, and the strain sensors are gaining attention due to their low cost, robustness and high reliability in their measurements and applications [25]. Therefore, it is feasible to use the $A_{\varepsilon}$ calculated by the strain signal as an index to estimate tire longitudinal force.

\section{ESTIMATION ALGORITHM OF A LONGITUDINAL FORCE}

\subsection{Finite-Element Tire Model}

Because the finite-element tire model established in this study was used only to identify the model parameters and verify the tire estimation algorithm, the model was constructed only considering the longitudinal pattern of the tire. The details of the tire model method were detailed previously in [26] and [27]. To improve the calculation accuracy of the contact area, the grid of the contact area was refined. When performing rotation to generate a three-dimensional grid, the rotation angle of the grid near the lower half of the tire's contact area was $1^{\circ}$ with 60 pitches, and the rotation angle over the remaining angle was $5^{\circ}$ with 60 pitches. The tire model is illustrated in Fig. 20 ; the total number of elements was 211,585 , and the total number of nodes was 227,546. The rim and road surface were defined as rigid analytical bodies, and the tire model was loaded and free-rolled. The tire loading process was implemented by applying road surface displacement.

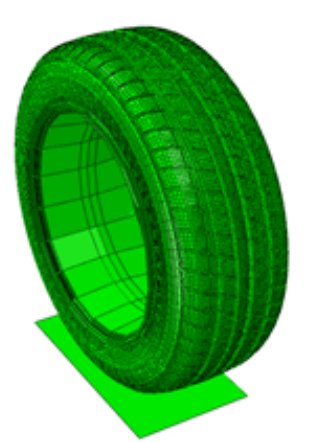

Fig. 20. Tire Finite element model

\subsection{Longitudinal Force Estimation Algorithm}

The overall framework of the intelligent tire longitudinal force estimation algorithm is displayed in Fig. 21. The input of the algorithm is the circumferential strain of the longitudinal centerline of the inner liner. In the signal processing part of the algorithm, $A_{\varepsilon}$ is calculated using the circumferential strain. In the output part of the algorithm, the linear regression model is used to output the longitudinal tire force.

The linear regression model uses a unary linear function, and the longitudinal force of the tire is considered to be a linear function of $A_{\varepsilon}$. The regression model is expressed as follows:

$$
F_{x}=a \cdot A_{\varepsilon}+b,
$$

where $a$ and $b$ are the fitting parameters to be identified.

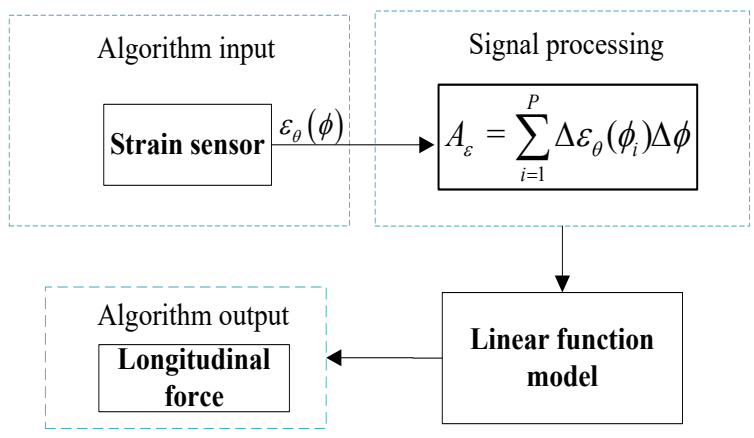

Fig. 21. Flowchart for longitudinal force estimation method

To conduct parameter identification for the linear function and verify the longitudinal force estimation algorithm, the longitudinal forces were simulated using a finite element model under different schemes, as shown in Table 2.

Table 2. Finite element simulation schemes

\begin{tabular}{ccc}
\hline Schemes & Vertical force [N] & Slip ratio [\%] \\
\hline 1 & 2000 & $3,6,9,12,20$ \\
\hline 2 & 2900 & $3,6,9,12,20$ \\
\hline 3 & 4000 & $3,6,9,12,20$ \\
\hline 4 & 5600 & $3,6,9,12,20$ \\
\hline
\end{tabular}

The finite-element simulation results provided the longitudinal forces corresponding to different slip rates. Schemes 1 and 3 were used to identify the fitting parameters of the linear function. The difference curve area $A_{\varepsilon}$ was calculated using the circumferential strain obtained through finite-element simulation. The following fitting parameters were obtained by linearly fitting the longitudinal force obtained from the finiteelement simulation: $a=1.96 \times 10^{6}, b=-28.35$. The longitudinal force values obtained using schemes 2 and 4 were used to verify the predicted results. Fig. 22 displays a comparison of the estimated and simulated longitudinal force. The results indicate that when the longitudinal force was less than $400 \mathrm{~N}$, the estimated error was $20 \%$ because the asymmetry of the strain was not considered when the longitudinal force was 
small. However, when the longitudinal force was greater than $400 \mathrm{~N}$, the estimated error decreased and is lower than $10 \%$. The main source of this error was the error in the strain simulation result caused by the finite-element mesh density. Because the nodal strain of Abaqus software was obtained from a geometric equation, the integral point strain was obtained from the extrapolation of the integral point stress. Moreover, different results are usually obtained when extrapolating the tensor of the integral point from multiple elements to the shared node. Therefore, the accuracy of the finite-element strain analysis results was relatively low, and the estimated longitudinal force exhibited a large error.

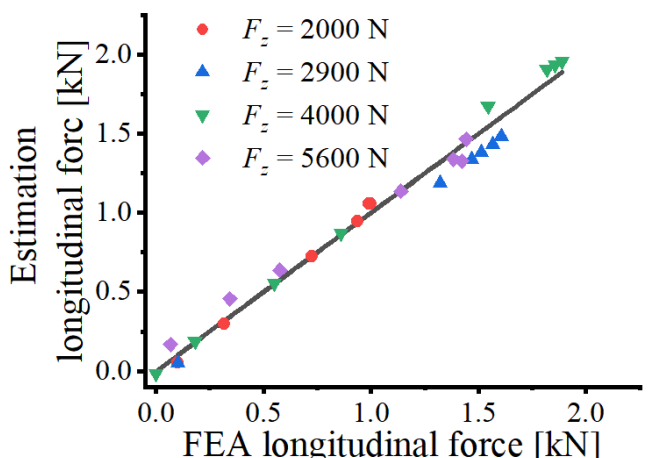

Fig. 22. Comparison of the longitudinal force estimated and simulated under different vertical force

\section{CONCLUSION}

This study established an algorithm for estimating the longitudinal tire force using the physics-based model. The following conclusions were drawn from this study:

1. A longitudinal dynamic tire model based on the flexible ring model and brush model was established, and the deformation and strain of the ring were obtained under different vertical and longitudinal forces. Due to the inextensibility of the high strength tire carcass, the radial deformations of tire carcass will not only result into tangential deformations but also results into circumferential stress and strain. The longitudinal force is the primary reason for the asymmetric distribution of the radial deformation and circumferential strain of the tire. Moreover, the greater the longitudinal force, the larger the asymmetry of the radial deformation and circumferential strain. The radial deformation difference $\Delta \omega$ and the circumferential strain difference $\Delta \varepsilon_{\theta}$ between the front and rear regions are proposed to describe the asymmetry quantitatively.

2. The difference curve area of radial deformation $A_{\omega}$ and the difference curve area of circumferential strain $A_{\varepsilon}$ are proposed to study the effects of the longitudinal force on the tire deformation and strain, and the results show that the $A_{\omega}$ and $A_{\varepsilon}$ have a strong linear relationship with the longitudinal tire forces under the different vertical loads and slip ratios. With these results, the $A_{\omega}$ and $A_{\varepsilon}$ can be used to estimate the longitudinal force acting on the tire.

3. Considering the low cost, robustness, and high reliability of the strain gauges in their measurements, tire circumferential strain information in this study is more suitable for estimating the longitudinal tire force. To establish the relationship between the circumferential strain and longitudinal force, a tire longitudinal force estimation algorithm was proposed, and the model parameters were also identified. From the simulation results under different vertical forces and slip ratios, the reliability of the proposed algorithm was validated. However, it is necessary to develop a practical intelligent tire based on strain. Experiments to reveal the relationship between tire strain and longitudinal force, and the determination of the strain sensor location, are objectives that remain for future study.

\section{ACKNOWLEDGEMENTS}

The author(s) disclosed receipt of the following financial support for the research, authorship, and/ or publication of this article: this work was jointly supported by the National Natural Science Foundation of China (Grant No. 52072156 and No. 51675240), Jiangsu provincial Six Talent Peaks (Grant No. JXQC-011), and Postdoctoral Foundation of China(2020M682269).

\section{NOMENCLATURES}

$R$ mean radius of ring, [m]

$\bar{R} \quad$ free radius of ring, [m]

$\Omega$ angular speed, [ $\mathrm{rad} / \mathrm{s}]$

$h$ the thickness of the ring, [m]

$K_{\omega}$ radial stiffness of sidewall, [N/m²]

$K_{v}$ tangential stiffness of sidewall

$K_{E t}$ radial stiffness of tread

$K_{G t}$ tangential stiffness of tread, [N/m²]

$w$ radial displacement of the neutral axis, [m]

$v$ tangential displacement of the neutral axis, [m] 
$c_{w}$ radial damping of sidewall, [Ns/m²]

$c_{v}$ tangential damping of sidewall, $\left[\mathrm{Ns} / \mathrm{m}^{2}\right]$

EI stiffness of the ring, $\left[\mathrm{Nm}^{2}\right]$

$\rho$ equivalent density of the ring $\left[\mathrm{kg} / \mathrm{m}^{3}\right]$

$b$ the width of the ring, [m]

$\tau$ depth of tire, [m]

$q_{w}$ radial external force on ring, [N]

$q_{v}$ tangential external force on ring, $[\mathrm{N}]$

$u_{r}$ displacement in radial direction, [m]

$u_{r}$ displacement in circumferential direction, [m]

$y$ distance from the neutral ring, [m]

\section{REFERENCES}

[1] Coppo, F., Pepe, G., Roveri, N., Carcaterra, A. (2017). A multisensing setup for the intelligent tire monitoring. Sensors, vol. 17, no. 3, art. ID 576, Dol:10.3390/s17030576.

[2] Du, X.B., Zhao, Y.Q., Wang, Q., Fu, H.X., Lin, F. (2019). Grounding characteristics of a non-pneumatic mechanical elastic tire in a rolling state with a camber angle. Strojniški vestnik - Journal of Mechanical Engineering, vol. 65, no. 5, p. 287-296, Dol:10.5545/sv-jme.2018.5845.

[3] Khaleghian, S., Emami, A., Taheri, S. (2017). A technical survey on tire-road friction estimation. Friction, vol. 5, no. 2, p. 123146, DOI:10.1007/s40544-017-0151-0.

[4] Lee, H., Taheri, S. (2017). Intelligent Tires? A review of tire characterization literature. IEEE Intelligent Transportation System Magazine, vol. 9, no. 2, p. 114-135, D0l:10.1109/ MITS.2017.2666584.

[5] Xiong, Y., Yang, X.G. (2018). A review on in-tire sensor systems for tire-road interaction studies. Sensors Review, vol. 38, no. 2, p. 231-238, DOI:10.1108/SR-07-2017-0132.

[6] Mendoza-Petit, M.F., García-Pozuelo, D., Díaz, V., Olatunbosun, 0. (2020). A strain-based intelligent tire to detect contact patch features for complex maneuvers. Sensors, vol. 20, no. 6, art. ID 1750, Dol:10.3390/s20061750.

[7] Ley-Rosas, J.J., Gonzalez-Jimenez, L.E., Loukianov, A.G., RuizDuarte, J.E. (2019). Observer based sliding mode controller for vehicles with roll dynamics. Journal of the Franklin Institute, vol. 356, no. 5, p. 2559-2581, D0l:10.1016/j. jfranklin.2018.11.031.

[8] Kunjunni, B., bin Zakaria, M.A., Majeed, A.P.P., Nasir, A.F.A., Hamid, U.Z.A. (2020). Effect of load distribution on longitudinal and lateral forces acting on each wheel of a compact electric vehicle. SN Applied Ences, vol. 2, art ID 244, D0l:10.1007/ s42452-020-1996-9.

[9] Yang, X., Olatunbosun, O., Ramos, D.G.P., Bolarinwa, E. (2013). Experimental investigation of tire dynamic strain characteristics for developing strain-based intelligent tire system. SAE International Journal of Passenger Cars-Mechanical Systems, vol. 6, no. 1, p. 97-108, DOl:10.4271/2013-01-0633.

[10] Matsuzaki, R., Todoroki, A. (2009). Intelligent tires for improved tire safety based on strain measurements, health monitoring of structural and biological systems 2009. Proceedings SPIE, vol. 7295, Dol:10.1117/12.815403.
[11] Zhao, J., Lu, Y.H, Zhu, B., Liu, S. (2018). Estimation algorithm for longitudinal and vertical forces of smart tire with accelerometer embedded. Automotive Engineering, vol. 40, no. 2, p. 137-142, Dol:10.19562/J.chinasae. qcgc.2018.02.003. (in Chinese)

[12] Zhu, X.L., Lu, D. (2017). A 'smart tire' for estimating the tire force and the extents of the influences of specific factors. Proceedings of the Institution of Mechanical Engineers, Part D: Journal of Automobile Engineering, vol. 231, no. 13, p. 1848-1857, Dol:10.1177/0954407016685633.

[13] Zhang, Y., Yi, J. (2014). Static tire/road stick-slip interactions: Analysis and experiments. IEEE/ASME Transactions on Mechatronics, vol. 19, no. 6, p. 1940-1950, D0l:10.1109/ TMECH.2013.2292872.

[14] Zhang, Y., Yi, J., Liu, T. (2013). Embedded flexible force sensor for in-situ tire-road interaction measurements. IEEE Sensors Journal, vol. 13, no. 5, p. 1756-1765, Dol:10.1109/ JSEN.2013.2241051.

[15] Yi, J., (2008). A piezo-sensor-based "smart tire" system for mobile robots and vehicles. IEEE/ASME Transactions on Mechatronics, vol. 13, no. 1, p. 95-103, D0l:10.1109/ TMECH.2007.915064.

[16] Garcia-Pozuelo, D., Yunta, J., Olatunbosun, O., Yang, X., Diaz, V. (2017). A strain-based method to estimate slip angle and tire working conditions for intelligent tires using fuzzy logic. Sensors, vol. 17, no. 4, p. 874-891, Dol:10.3390/s17040874.

[17] Xiong, Y., Tuononen, A. (2015). The in-plane deformation of a tire carcass: Analysis and measurement. Case Studies in Mechanical Systems and Signal Processing, vol. 2, p. 12-18, Dol:10.1016/j.csmssp.2015.09.001.

[18] Garcia-Pozuelo, D., Olatunbosun, O., Strano, S., Terzo, M. (2019). Areal-time physical model for strain-based intelligent tires. Sensors and Actuators, A: Physical, vol. 288, p. 1-9, DOl:10.1016/j.sna.2018.12.010.

[19] Wei, Y.T., Nasdala, L., Rothert, H. (2009). Analysis of forced transient response for rotating tires using ref models. Journal of Sound and Vibration, vol. 320, no. 1-2, p. 145-162, DOI:10.1016/j.jsv.2008.07.007.

[20] Kim, S.J., Savkoor, A.R. (1996). The contact problem of inplane rolling of tires on a flat road. Vehicle System Dynamics, vol. 27, p. 189-206, DOl:10.1080/00423119608969654.

[21] Liu, Z., Zhou, F.Q., Oertel, C., Wei, Y.T. (2017). Threedimensional vibration of a ring with a noncircular cross-section on an elastic foundation. Proceedings of the Institution of Mechanical Engineers, Part C: Journal of Mechanical Engineering Science, vol. 232, no. 13, p. 2381-2393, DOI:10.1177/0954406217720823.

[22] Guo, K.H. (2016). Untire:Untire tire model. Journal of Mechanical Engineering, vol. 52, no. 12, p. 90-99, DOI:10.3901/JME.2016.12.090. (In Chinese)

[23] Nishihara, O., Masahiko, K. (2011). Estimation of road friction coefficient based on the brush model. Journal of Dynamic Systems, Measurement, and Control, vol. 133, no. 4, DOI:10.1115/1.4003266.

[24] Han, T. (2020). Study on tire force estimation method of intelligent tires by using flexible ring model. MSc Thesis, Jiangsu University. (in Chinese). 
[25] Garcia-Pozuelo, D., Olatunbosun, O., Yunta, J., Yang, X., Diaz, V. (2017). A novel strain-based method to estimate tire conditions using fuzzy logic for intelligent tires. Sensors, vol.17, no. 2, p. 350-366, D0l:10.3390/s17020350.

[26] Zhou, H.-C., Wang, G.-L., Yang, J., Xue, K.-X. (2015). Numerical simulation of the effect of bionic v-riblet non-smooth surface on tire anti-hydroplaning. Journal of Central South University, vol. 22, p. 3900-3908, D0I:10.1007/s11771-015-2934-7.

[27] Yang, J., Wang, G.L., Wan, Z.J., Liang, C., Zhou, H.C. (2016). Non-natural equilibrium contour design for radial tire and its influence on tire performance. International Journal of Automotive Technology, vol. 17, no. 4, p. 639-649, DOI:10.1007/s12239-016-0063-4. 